\title{
Multiple levels of visual object constancy revealed by event-related fMRI of repetition priming
}

\author{
P. Vuilleumier ${ }^{1}$, R. N. Henson ${ }^{1,2}$, J. Driver ${ }^{1}$ and R. J. Dolan ${ }^{2,3}$ \\ ${ }^{1}$ Institute of Cognitive Neuroscience, University College London, Alexandra House, 17 Queen Square, London WC1N 3AR, UK \\ ${ }^{2}$ Functional Imaging Laboratory, Wellcome Department of Cognitive Neurology, 12 Queen Square, London WC1N 3BG, UK \\ ${ }^{3}$ Royal Free Hospital and University College School of Medicine, Roland Hill Street, London NW3 2PF, UK \\ Correspondence should be addressed to P.V. (p.vuilleumier@ucl.ac.uk)
}

Published online: 22 April 2002, DOI: 10.1038/nn839

\begin{abstract}
We conducted two event-related functional magnetic resonance imaging (fMRI) experiments to investigate the neural substrates of visual object recognition in humans. We used a repetitionpriming method with visual stimuli recurring at unpredictable intervals, either with the same appearance or with changes in size, viewpoint or exemplar. Lateral occipital and posterior inferior temporal cortex showed lower activity for repetitions of both real and non-sense objects; fusiform and left inferior frontal regions showed decreases for repetitions of only real objects. Repetition of different exemplars with the same name affected only the left inferior frontal cortex. Crucially, priming-induced decreases in activity of the right fusiform cortex depended on whether the threedimensional objects were repeated with the same viewpoint, regardless of whether retinal image size changed; left fusiform decreases were independent of both viewpoint and size. These data show that dissociable subsystems in ventral visual cortex maintain distinct view-dependent and view-invariant object representations.
\end{abstract}

The human visual system has a remarkable capacity to recognize thousands of objects, despite changes in location, distance, viewpoint and illumination. How the brain achieves this 'object constancy' is largely unknown ${ }^{1-5}$. Visual recognition may involve several stages, from extracting shape information based on cues such as edges and textures, to matching this information with memory representations. The precise nature of such object representations is currently under dispute ${ }^{3-5}$.

Some theories hold that the visual system builds abstract threedimensional (3D) models of objects from two-dimensional (2D) retinal images ${ }^{6}$, and stores visual information independent of momentary viewing parameters such as orientation or scale ${ }^{1}$. Others suggest that recognition relies primarily on view-specific processes, and is based on past encounters with an object ${ }^{5,7}$. Psychophysical $^{7-9}$ and neuropsychological ${ }^{10-12}$ findings support both explanations, resulting in a 'view-specific recognition' versus 'viewindependent recognition' split ${ }^{4,5,9}$. Furthermore, single-cell recordings from inferior temporal cortex in monkeys have revealed some visual neurons that respond to a stimulus across different sizes and views ${ }^{13,14}$, but a greater number that respond to a stimulus only when presented at a specific view or size $e^{15,16}$.

We used event-related fMRI during a repetition-priming task to identify neural subsystems that process visual objects at distinct stages of representation, with particular interest in brain regions showing differential sensitivity to changes in viewpoint and/or size. Object invariance was tested by comparing the priming-related fMRI response to a repeated stimulus (with the same or different appearance) with those responses to the first presentation of the stimulus. Behaviorally, perceptual priming improves performance on various object recognition tasks, with or without explicit recollection of the initial viewing ${ }^{17}$. Studies of this learning effect report that priming occurs across changes in object position ${ }^{18}$, size $^{19,20}$, color $^{21}$ and even viewpoint when crucial parts of the image are preserved ${ }^{2,9}$. Recent findings show, however, that recognition is best with repetition of the same viewpoint ${ }^{3-5,7}$; this has been interpreted as evidence for view-based representations. If it is the case that adjacent columns of neurons in temporal cortex each code for distinct views, pooling different columns of neurons to interpolate between these representations might then underlie apparent view-invariant performance ${ }^{3,5}$.

Single-cell recordings in monkeys ${ }^{13,22}$ and functional imaging in humans ${ }^{17}$ have shown that for neurons tuned to particular stimulus attributes, priming decreases the neural response to repeated presentations of a stimulus as compared with the initial presentation. Positron emission tomography (PET) and fMRI studies have shown such priming-related decreases in temporal cortex for repeated words ${ }^{23,24}$, faces ${ }^{25}$ and objects ${ }^{26-28}$. In most studies, the same $e^{25,26}$ or part of the same $e^{23,27}$ stimulus was repeated. Other experiments have compared conditions such as identical object versus different exemplars sharing the same name ${ }^{28}$, nameable versus non-nameable line drawings ${ }^{29}$ and structurally possible versus impossible objects ${ }^{30}$.

Repetition effects in event-related fMRI are useful for determining the critical properties to which neurons respond, even when populations with different selectivity overlap in the same region. Thus, if neurons in neighboring columns code for distinct views of 
a Real objects:
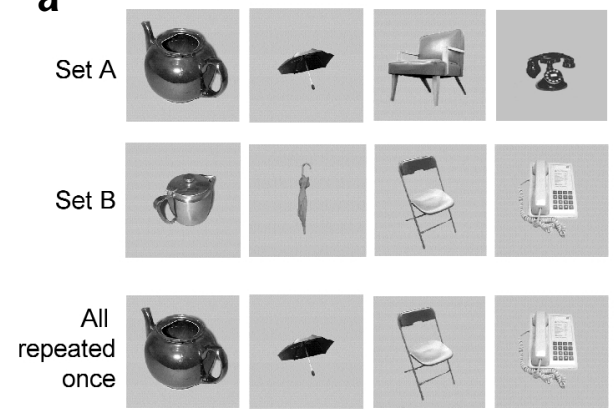

Repeated again:

b

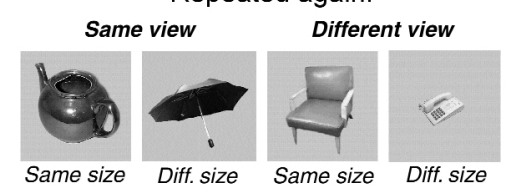

Non-objects:
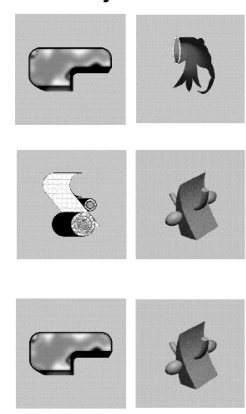

New:

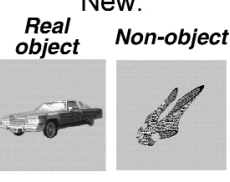

Fig. I. Examples of stimuli and schematic illustration of experimental design. (a) In experiment I, real and non-sense objects were shown twice each, in identical form. Real object categories, such as 'chairs', had two exemplars each. This resulted in six trial types: a particular item from a real object category $\left(\mathrm{RO}_{\mathrm{A}}\right)$, another item from that category $\left(\mathrm{RO}_{B}\right)$ or a non-sense object (NO), each presented either for the first or second time (for example, $\mathrm{RO}_{\mathrm{AI}}$ versus $R O_{A 2}$ ). (b) In experiment 2, all real objects from the first experiment were shown a third time with either the same or a different viewpoint, and either the same or a different size, intermingled with new objects and new non-sense objects. This resulted in six trial types: real objects repeated with same view and same size $\left(\mathrm{V}_{s} \mathrm{~S}_{s}\right)$, same view but different size $\left(\mathrm{V}_{s} \mathrm{~S}_{d}\right)$, same size but different view $\left(V_{d} S_{s}\right)$, different view and different size $\left(V_{d} S_{d}\right)$; new real objects $\left(\mathrm{RO}_{\text {new }}\right)$; and new non-sense objects $\left(\mathrm{NO}_{\text {new }}\right)$. The same critical object parts were kept visible across viewpoint changes. An equal number of items from set $A$ and set $B$ were distributed within each repeated condition.

Participants classified each item as a real or non-sense object the same object (as proposed by some theories of view-specific recognition ${ }^{5}$ ), then regional activity should be reduced by repeating an object with the same view, but not by repeating an object with a different view. A similar logic was applied in recent functional imaging work ${ }^{31-33}$ to examine the effects of various shape or object transformations (retinal position, size, rotation, illumination or partial occlusion). One experimental design varies these parameters across immediate repetition trial pairs ${ }^{31,32}$; another compares blocks of repeated identical images with blocks of changing images ${ }^{33}$. Although this 'fMRI adaptation' approach has proved useful ${ }^{34}$, data from immediate repetition studies may reflect anticipated recurrence or novelty/mismatch effects on attention ${ }^{35,36}$, rather than priming effects per se. Moreover, neural adaptation during repetition of the same image may not relate to the long-term priming effects found in behavioral object recognition tasks.

We therefore examined the fMRI correlates of priming when same or different images recurred randomly after an unpredictable number of intervening trials, so that subjects would not come to expect repetitions or particular changes in object appearance. With this design we also were able to assess whether behavioral facilitation was concomitant with fMRI repetition effects. By systematically manipulating repetition, we delineated neural systems with differential sensitivity to specific object attributes (size, view or name). Our main findings indicate view-independent priming in left fusiform cortex, and view-dependent priming in right fusiform cortex.

\section{REsULTS}

\section{Experiment 1}

In the first experiment, real and non-sense 3D objects were each shown twice in a pseudo-random order (Fig. 1a; see Methods). All real objects included two distinct items from the same category with the same name (any object from set B occurring later than a same-name object from set A). The primary goal of this experiment was to give participants exposure to objects that would be subsequently repeated as primed stimuli in experiment 2. A secondary goal was to investigate (i) effects of stimulus repetition, including effects of repeating visually-identical images of real objects (that match pre-existing memory representations) or nonsense objects (that presumably do not match pre-existing representations of the whole object); and (ii) effects of repeating meaningful objects that shared the same name but were visually distinct (different exemplars of the same category).

by key-presses, using the right hand. Performance revealed few errors (mean, 2.1\%) and reliable behavioral priming (Table 1). Repeated-measures analysis of variance (ANOVA) showed that reaction times (RTs) were faster for real objects than for nonsense objects $\left(F_{1,11}=22.3, P<0.001\right)$, and faster on repeated than on first occurrences for both stimulus types $\left(F_{1,11}=28.7, P<\right.$ $0.001)$. There was a significant interaction between stimulus type and repetition $\left(F_{1,11}=5.8, P=0.03\right)$, indicating greater priming for real objects than for non-sense objects. Repetition of objects with the same name produced a non-significant advantage for the first occurrence of items in set $\mathrm{B}$, as compared to the first occurrence of similar exemplars in set A (Table 1; paired $t$-test, $t_{11}=1.11, P=0.14$, one-tailed).

\section{Repetition effects in fMRI}

We first determined regions showing common repetition decreases for both real $(\mathrm{RO})$ and non-sense $(\mathrm{NO})$ objects (a conjunction of $\left[\mathrm{RO}_{\mathrm{A} 1}+\mathrm{RO}_{\mathrm{B} 1}>\mathrm{RO}_{\mathrm{A} 2}+\mathrm{RO}_{\mathrm{B} 2}\right]$ and $\left.\left[\mathrm{NO}_{1}>\mathrm{NO}_{2}\right]\right)^{37}$. Such effects were found in bilateral inferior temporal gyri, extending medially into posterior fusiform cortex and laterally into right inferior occipital gyrus (Table 2; Fig. 2a, b, and c). These regions correspond to those previously described as the lateral occipital

\section{Table I. Response times on object decision task during fMRI scanning.}

\begin{tabular}{lll}
\hline Trial types & & Mean RTs (ms) \\
Experiment I & & \\
Real objects & Set A, first presentation & 735 \\
& Set B, first presentation & 721 \\
& Set A, repeated & 671 \\
& Set B, repeated & 669 \\
Non-sense objects & First presentation & 771 \\
& Repeated & 731 \\
Experiment 2 & & \\
Repeated real objects & Same view + same size & 616 \\
& Same view + different size & 633 \\
& Different view + same size & 658 \\
& Different view + different size & 654 \\
New real objects & & 685 \\
Non-sense objects & & 758 \\
\hline
\end{tabular}


complex (LOC), known to respond better to structured objects than to unstructured textures ${ }^{34,38}$.

Brain regions showing reduced activity with repetition versus first presentation of real objects $\left(\mathrm{RO}_{\mathrm{A} 1}+\mathrm{RO}_{\mathrm{B} 1}\right.$ $>\mathrm{RO}_{\mathrm{A} 2}+\mathrm{RO}_{\mathrm{B} 2}$ ) included bilateral fusiform and inferior temporo-occipital cortex, as well as left posterior inferior frontal cortex (Table 2). Fusiform responses extended from the temporo-occipital sulcus posteriorly to the collateral sulcus anteriorly (Fig. 2a and d). The same comparison restricted to non-sense objects $\left(\mathrm{NO}_{1}>\mathrm{NO}_{2}\right)$ showed significant repetition effects in inferior temporal cortex alone (Table 2). At a lower statistical threshold, weak decreases for repeated non-sense objects were additionally seen only in lateral occipital cortex (peak Talairach $x, y, z$ coordinates, $42,-87,-6$ and $-48,-72,-3$; $Z=2.89$ and $2.73 ; P \leq 0.003$ uncorrected) and right posterior fusiform gyrus $(42,-54,-21 ; Z=2.75 ; P=0.003)$. Thus, repetition of meaningful objects affected larger and more anterior areas as compared with the meaningless, though structurally possible, non-sense objects (Fig. 2). This was confirmed by testing for regions showing a significant interaction between stimulus type and repetition: namely, greater repetition decreases for real than for non-sense objects $\left(\left[\mathrm{RO}_{\mathrm{A} 1}+\mathrm{RO}_{\mathrm{B} 1}\right]-\left[\mathrm{RO}_{\mathrm{A} 2}+\mathrm{RO}_{\mathrm{B} 2}\right]\right.$ $\left.>\left[\mathrm{NO}_{1}-\mathrm{NO}_{2}\right]\right)$. Areas showing such an effect were found in fusiform cortex, predominantly on the left (Fig. 2g), as well as in left frontal cortex (Table 2). The same results were obtained when testing repetition effects for an equal number of real and non-sense objects (rather than twice as many real objects), using two different randomly selected subsets of real objects for each subject. Overall, these fMRI results paralleled our behavioral findings of greater priming for real objects than for non-sense objects. No voxel showed repetition decreases that were significantly greater for non-sense objects.

Notably, fusiform regions were unaffected by repetition of different exemplars from the same category with the same name (Fig. $2 \mathrm{~h}$ and $\mathbf{i}$ ), indicating that selective repetition effects for real objects involved visual representations, not lexico-semantic processes (such as mentally naming recognized stimuli, which subjects were not instructed to do). When the first occurrence of nameable objects was directly compared to the subsequent repetitions of any items with the same name $\left(\mathrm{RO}_{\mathrm{Al}}>\mathrm{RO}_{\mathrm{B} 1}+\right.$ $\mathrm{RO}_{\mathrm{A} 2}+\mathrm{RO}_{\mathrm{B} 2}$ ), significant decreases specific to lexico-semantic repetition were found in left inferior frontal gyrus alone (Table 2; Fig. $2 \mathbf{j}$ and $\mathbf{k}$ ). This corroborates previous studies of semantic priming 24,39 .

Some regions were activated more by real than by non-sense objects, independent of repetition $\left(\left[\mathrm{RO}_{\mathrm{A} 1}+\mathrm{RO}_{\mathrm{B} 1}+\mathrm{RO}_{\mathrm{A} 2}+\mathrm{RO}_{\mathrm{B} 2}\right]\right.$ $\left.>\left[\mathrm{NO}_{1}+\mathrm{NO}_{2}\right]\right)$. These included bilateral anterior fusiform, left posterior parietal and left parahippocampal cortex (Table 3 ). No region showed greater activation for non-sense than for real objects.

\section{Correlation with reaction times}

As RTs differed for each task condition, we further analyzed the imaging data to find brain areas where activity varied in direct proportion to the RT to each stimulus, regardless of trial type. Such RT effects were primarily found in premotor regions of lateral frontal $(45,27,-12 ; Z=9.30 ;-30,-24,-12 ; Z=8.68 ; 51,21,24 ; Z$ $=8.63)$ and medial frontal cortex $(3,27,42 ; Z=8.84$; all $P<0.05$ corrected). Additional effects were found in fusiform areas $(-48$, $-57,-21, Z=6.32 ; 48,-63,-18, Z=6.05 ; P<0.05)$, consistent with

\begin{tabular}{|c|c|c|c|c|c|c|}
\hline \multirow[t]{2}{*}{ Side } & \multirow[t]{2}{*}{ Brain areas } & \multicolumn{3}{|c|}{ Coordinates } & \multirow[t]{2}{*}{ Z-score } & \\
\hline & & $x$ & $y$ & $z$ & & \\
\hline \multicolumn{7}{|c|}{ Repetition decrease for real objects } \\
\hline $\mathrm{L}$ & Posterior fusiform gyrus & -45 & -60 & -18 & 5.66 & \\
\hline $\mathrm{L}$ & Anterior fusiform gyrus & -36 & -39 & -27 & 5.35 & \\
\hline $\mathrm{L}$ & Inferior temporal gyrus & -42 & -72 & -12 & 4.67 & \\
\hline $\mathrm{L}$ & Lateral/inferior occipital gyrus & -27 & -75 & 33 & 3.41 & * \\
\hline $\mathrm{R}$ & Posterior fusiform gyrus & 42 & -57 & -18 & 5.14 & \\
\hline $\mathrm{R}$ & Inferior temporal gyrus & 48 & -69 & -12 & 4.49 & \\
\hline $\mathrm{R}$ & Anterior fusiform gyrus & 39 & -36 & -27 & 3.57 & * \\
\hline $\mathrm{R}$ & Lateral/inferior occipital gyrus & 42 & -84 & 6 & 4.09 & \\
\hline $\mathrm{L}$ & Posterior inferior frontal gyrus & -39 & 6 & 27 & 4.63 & \\
\hline L & $\begin{array}{l}\text { Frontal operculum } \\
\text { (pars triangularis) }\end{array}$ & -48 & 36 & 12 & 3.74 & \\
\hline \multicolumn{7}{|c|}{ Repetition decrease for non-objects } \\
\hline L & Inferior temporal gyrus & -48 & -66 & -15 & 3.04 & * \\
\hline \multicolumn{7}{|c|}{ Interaction: repetition decrease for real objects > for non-objects } \\
\hline L & Anterior fusiform gyrus & -33 & -36 & -27 & 4.02 & \\
\hline $\mathrm{L}$ & Posterior fusiform gyrus & -33 & -54 & -15 & 3.42 & * \\
\hline $\mathrm{L}$ & Inferior temporal gyrus & -48 & -54 & -21 & 3.74 & \\
\hline $\mathrm{L}$ & Posterior inferior frontal gyrus & -39 & 6 & 27 & 3.93 & \\
\hline $\mathrm{R}$ & Posterior fusiform gyrus & 48 & -57 & -21 & 3.39 & * \\
\hline \multicolumn{7}{|c|}{ Repetition decrease common for objects and non-objects } \\
\hline \multirow[t]{2}{*}{ L } & Inferior temporal gyrus & -48 & -66 & -15 & 4.64 & \\
\hline & /posterior fusiform gyrus & -39 & -57 & -24 & 3.78 & \\
\hline \multirow[t]{2}{*}{$R$} & Inferior temporal gyrus & 30 & -51 & -24 & 4.35 & \\
\hline & /posterior fusiform gyrus & 42 & -54 & -21 & 4.25 & \\
\hline \multirow[t]{2}{*}{$\mathrm{R}$} & Lateral/inferior occipital gyrus & 42 & -81 & 12 & 4.11 & \\
\hline & & 45 & -84 & -3 & 4.05 & \\
\hline $\mathrm{L}$ & Lateral/inferior occipital gyrus & -45 & -75 & -6 & 3.65 & \\
\hline
\end{tabular}

Semantic repetition: first > other objects with same name L Frontal operculum

$\begin{array}{lllll}\text { (pars triangularis) } & -48 & 36 & 12 & 3.60\end{array}$

$\overline{\text { Activation peaks significant at } P<0.05 \text { corrected for multiple comparisons, except for }}$ $* P<0.00$ I uncorrected.

the mean RT decreases associated with visual perceptual priming. Taking RT effects into account, different trial types produced distinct responses in visual cortex, with specific effects in bilateral fusiform regions due to stimulus repetition (first $>$ second presentation, $-45,-66,-15, Z=5.34 ; 42,-57,-18, Z=4.92$ ) and stimulus type (real $>$ non-sense objects, $-42-51-27, Z=4.99 ; 39,-54,-21$, $Z=4.97$; all $P<0.05$ corrected).

\section{Experiment 2}

After a 3-4 minute break, subjects began a second task-the critical test for invariance in priming across changes in object appearance. All real objects presented during experiment 1 were shown once again, this time with size (same or different) and viewpoint (same or different) manipulated as compared with the first presentation, in a systematic $2 \times 2$ factorial design (Fig. 1b). Repeated, or 'old', objects were randomly intermingled with new real objects and new non-sense objects.

Participants again performed an object-decision task, making few errors (mean 1.5\%) and showing reliable behavioral priming (Table 1). There was a significant effect of stimulus type (repeated, 
Fig. 2. Repetition priming effects in experiment I. Group results are shown on a single-subject MRI scan (activated voxels $P<0.00 I$ ) and plotted across conditions for main peaks of activity in homologous areas of right and left hemispheres (mean percent of signal change from baseline \pm s.e.m). (a) Lateral occipital and inferior temporal gyri showing common repetition priming for real and non-sense objects. (b, c) Overall activity and priming effects in these regions were similar for real and non-sense objects. (d) Bilateral posterior fusiform and left frontal regions show priming for real objects. (e, f) Posterior fusiform responses were greater with real objects, and smaller with non-sense objects. (g) Left anterior fusiform regions show priming for real but not for non-sense objects. $(h, i)$ Overall activity and priming effects in anterior fusiform were selective for real objects, particularly on the left side. (j, k) Left inferior frontal regions (in the vicinity of Broca's region) showing semantic repetition priming on first presentation of objects (set B) from the same category and with the same name as other previously seen objects (set $A$ ), in addition to itemspecific priming within each object set. Talairach coordinates $(x, y, z)$ shown above each graph.

new or non-sense objects; $F_{2,10}=$ 17.5, $P<0.001$ ), with faster RTs to repeated than to new objects $\left(F_{1,10}=46.8, P<0.001\right)$. Further analysis restricted to repeated objects revealed an RT cost for

a

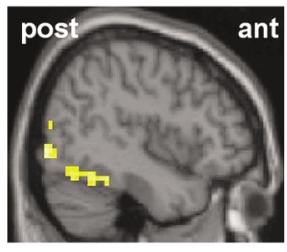

d Real objects
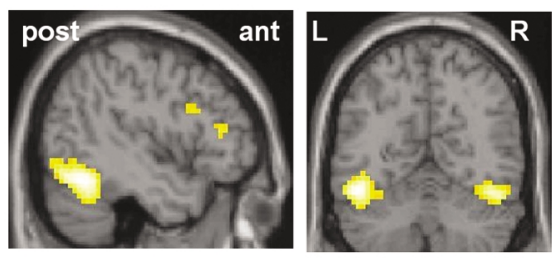

\section{9}

Real > non-sense objects

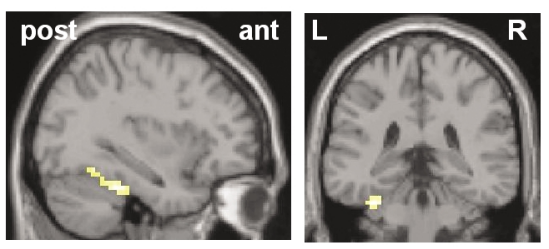

j Same object category
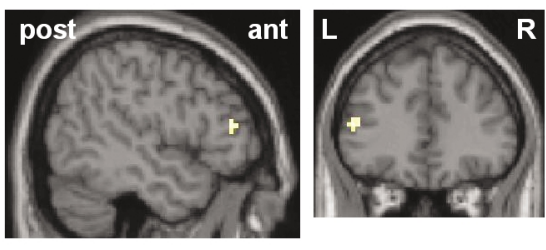
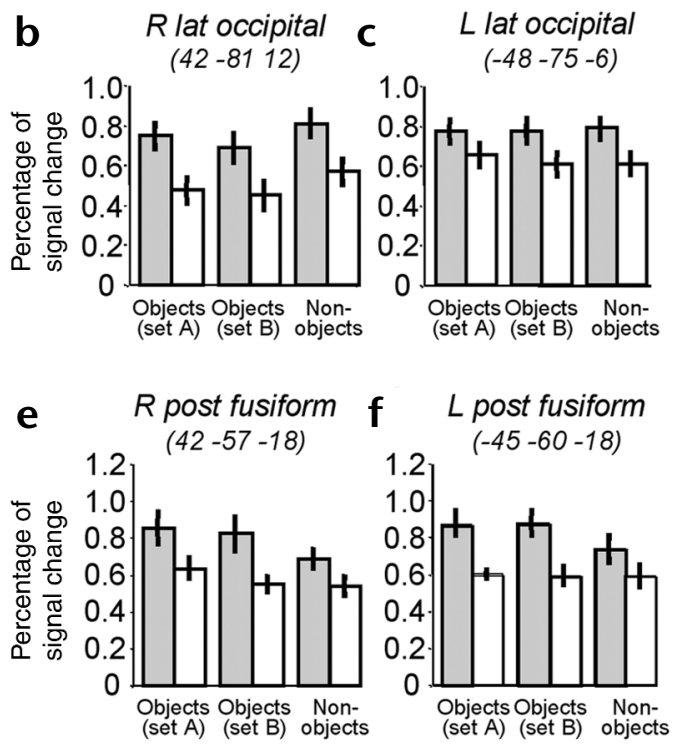

h

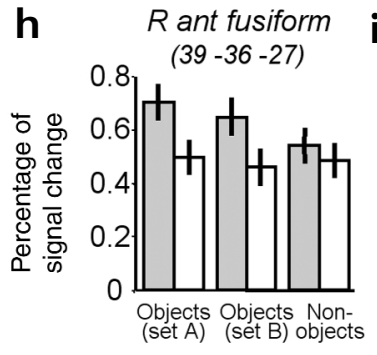

i $\quad L$ ant fusiform

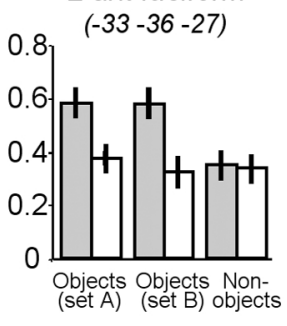

k

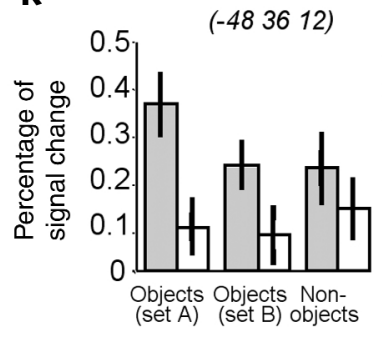

changing viewpoint $\left(F_{1,10}=5.3\right.$, $P=0.044)$ but not for changing size $\left(F_{1,10}=0.5\right)$, with no interaction between size and viewpoint $\left(F_{1,10}=0.7\right)$. Notably, RTs to objects repeated with changes in both view and size were still faster than those to new objects $\left(t_{10}=3.5, P=0.005\right)$, indicating some 'object constancy' in priming.

\section{Repetition effects in fMRI}

A main effect of object repetition, with decreased activity for all repeated versus new real objects, pooling over all views and sizes, was found bilaterally in anterior and posterior regions of fusiform cortex (Table 4).

Our factorial manipulation of size and view across repetition (Fig. 1b) enabled us not only to test for neural systems maintaining size- or view-specific representations, but also to distinguish between these two different forms of image specificity ${ }^{8,19}$. This design allowed us to test whether view- and size-specific effects were independent or interacted (if view-specific codes were also size-specific, as suggested by some models ${ }^{4,16,40}$ ) in any brain regions. All analyses contrasting the repetition of objects as a function of same versus different size (for example, 'old' objects repeated under the same view, $\mathrm{V}_{s} \mathrm{~S}_{\mathrm{d}}>\mathrm{V}_{\mathrm{s}} \mathrm{S}_{\mathrm{s}}$ ) and all tests for repetition effects unique to preserving both size and view (namely, regions showing an interaction of size and view) yielded no significant differences, even at liberal statistical thresholds. These findings support the idea that our fMRI repetition priming effects arise in object representations that generalize across different retinal-image sizes ${ }^{20,31}$.

Brain regions coding objects in a view-specific manner were identified by the main effect of changing viewpoint-comparing novel versus same views of 'old' repeated objects, regardless of whether they had the same or different size $\left(V_{d} S_{s}+V_{d} S_{d}>V_{s} S_{s}+\right.$ $V_{s} S_{d}$ ). The absence of size effects ensured that any viewpoint effects in this comparison did not result from other basic changes in the retinal image. Such view-dependent (but size-independent) repetition decreases were observed in right fusiform gyrus (Table 4; Fig. 3a, b, and e). There were also significant view-dependent repetition decreases in right posterior parietal cortex and right superior lateral occipital cortex. However, these parieto-occipital regions showed a different pattern than the fusiform. Right fusiform responses to same views (irrespective of size) decreased, but those 


\begin{tabular}{|c|c|c|c|c|c|c|}
\hline \multirow[t]{2}{*}{ Side } & \multirow[t]{2}{*}{ Brain areas } & \multicolumn{3}{|c|}{ Coordinates } & \multicolumn{2}{|l|}{ Z-score } \\
\hline & & $x$ & y & $z$ & & \\
\hline \multicolumn{7}{|c|}{ Experiment I: All real objects > all non-objects } \\
\hline L & $\begin{array}{l}\text { Posterior parietal cortex } \\
\text { (angular gyrus) }\end{array}$ & -39 & -81 & 27 & 4.25 & \\
\hline $\mathrm{L}$ & $\begin{array}{l}\text { Posterior parietal cortex } \\
\text { (angular gyrus) }\end{array}$ & -30 & -84 & 33 & 3.67 & \\
\hline $\mathrm{L}$ & Posterior fusiform gyrus & -42 & -51 & -27 & 4.05 & \\
\hline $\mathrm{L}$ & Anterior fusiform gyrus & -27 & -45 & -21 & 3.63 & $*$ \\
\hline R & Posterior fusiform gyrus & 39 & -54 & -24 & 3.86 & \\
\hline $\mathrm{L}$ & Parahippocampal gyrus & -9 & -54 & 3 & 3.76 & $*$ \\
\hline $\mathrm{L}$ & Cuneus & -3 & -81 & 15 & 3.58 & $*$ \\
\hline \multicolumn{7}{|c|}{ Experiment 2: New real objects $>$ new non-objects } \\
\hline L & $\begin{array}{l}\text { Posterior parietal cortex } \\
\text { (angular gyrus) }\end{array}$ & -36 & -81 & 24 & 3.87 & $*$ \\
\hline $\mathrm{R}$ & Fusiform gyrus & 42 & -51 & -18 & 3.48 & $*$ \\
\hline L & $\begin{array}{l}\text { Anterior inferior } \\
\text { frontal cortex }\end{array}$ & -42 & 33 & 9 & 3.43 & $*$ \\
\hline $\mathrm{L}$ & Parahippocampal gyrus & -9 & -51 & 3 & 3.28 & $*$ \\
\hline $\mathrm{L}$ & Fusiform gyrus & -39 & -63 & -15 & 3.24 & $*$ \\
\hline $\mathrm{L}$ & Anterior temporal lobe & -39 & 18 & -33 & 3.20 & * \\
\hline
\end{tabular}

Activation peaks significant at $P<0.05$ corrected for multiple comparisons, except for $* P<0.001$ uncorrected.

to novel views of 'old' objects and entirely new objects did not (Fig. 3b and e). By contrast, right parietal and superior occipital responses showed not only a moderate decrease to same views, but also a greater activation by new views of 'old' objects than by entirely new objects (Fig. 3g and h). View-dependent effects in parietooccipital regions may therefore implicate mechanisms different from standard repetition priming (possibly some mental rotation processes engaged by recognizing known items in a novel orientation $^{10,12}$ or object-directed tacit motor processes).

Finally, we identified areas showing repetition effects across the most extreme changes in visual appearance for the same object, by comparing 'old' objects repeated with both a different size and a different view against entirely new objects $\left(\mathrm{V}_{\mathrm{d}} \mathrm{S}_{\mathrm{d}}<\mathrm{RO}_{\text {new }}\right)$. Recall that behavioral priming was found even for this comparison. In the fMRI data, this contrast revealed a significant effect in left fusiform gyrus alone (Table 4; Fig. 3d). A greater view dependency in the right hemisphere, compared with the greater generalization across changed viewpoint in the left hemisphere (Fig. $3 \mathrm{c}$ and $\mathrm{f}$ ), was confirmed by an ANOVA on fusiform activity across all conditions showing a view $\times$ hemisphere interaction $\left(F_{1,10}=5.55, P=0.04\right)$. There was no main effect of size or any other interaction.

Thus, whereas the right fusiform showed view-dependent but size-independent priming, the left fusiform showed generalization across views and sizes. These findings were corroborated by single-subject analyses in which we first identified regions showing maximal repetition effects in the independent dataset of experiment 1 , and then examined fMRI responses in the same voxels for the critical conditions of experiment 2. For each participant, we selected bilateral peaks in the lateral occipital (mean $x, y, z \pm 45,-78,09$ ), posterior fusiform (mean \pm 40 , $-56,-22)$, and anterior fusiform cortex (mean $\pm 32,-38,-27)$.
These analyses again showed view-dependent effects only in the right hemisphere (see also Supplementary Fig. 1 online), with a significant view $\times$ hemisphere interaction in lateral occipital cortex $\left(F_{1,10}=7.2, P<0.05\right)$, posterior fusiform $\left(F_{1,10}=11.6\right.$, $P<0.01)$ and anterior fusiform $\left(F_{1,10}=18.7, P<0.01\right)$. The triple interaction of view $\times$ hemisphere $\times$ region was also significant $\left(F_{1,10}=4.05, P<0.05\right)$. Objects repeated with a different view produced significant decreases in activity (compared to new objects) in the left hemisphere, in posterior and anterior fusiform $\left(F_{1,10}=13.4\right.$ and 9.1, respectively, $\left.P<0.05\right)$, but not in lateral occipital cortex $\left(F_{1,10}=1.3\right)$.

As in experiment 1, comparing real versus non-sense objects $\left(\mathrm{RO}_{\text {new }}>\mathrm{NO}_{\text {new }}\right)$ revealed activations in right fusiform, left posterior parietal and left parahippocampal cortex (Table 3). Again, no region in occipito-temporal cortex was activated more by nonsense than by real objects.

\section{Responses specific to retinal size}

To verify that our manipulation of object size was effective, we carried out an additional analysis comparing large versus small objects, pooling across repetition and type (real or non-sense). This revealed marked activity increases in retinotopic areas around the calcarine sulcus (primary visual cortex; Table 5 and Fig. 4). The opposite contrast (small > large) found no significant voxel. None of these retinotopic areas showed a main effect of repetition $(Z \leq 0.55$ in experiment $1, Z \leq 0.16$ in experiment 2 , $P \geq 29$ uncorrected) or of size changes across repetition in experiment 2 (all $Z \leq 0.93, P \geq 0.17$ ).

\section{Discussion}

These results point to dissociable and lateralized neural components in the ventral visual system, each associated with perceptual learning at a different stage of object representation (Fig. 5). Bilateral regions in lateral occipital and posterior inferior temporal cortex showed repetition-related decreases independent of previous object knowledge (for both non-sense and real items). More rostral and ventral regions in the posterior

\begin{tabular}{|c|c|c|c|c|c|c|}
\hline \multirow[t]{2}{*}{ Side } & \multirow[t]{2}{*}{ Brain areas } & \multicolumn{3}{|c|}{ Coordinates } & \multicolumn{2}{|l|}{$Z$-score } \\
\hline & & $x$ & $y$ & $z$ & & \\
\hline \multicolumn{7}{|c|}{ All repeated $>$ new objects } \\
\hline $\mathrm{R}$ & Posterior fusiform gyrus & 48 & -51 & -21 & 5.5 & \\
\hline R & Posterior fusiform gyrus & 45 & -63 & -18 & 4.32 & \\
\hline $\mathrm{R}$ & Anterior fusiform gyrus & 33 & -36 & -30 & 3.8 & \\
\hline $\mathrm{L}$ & Posterior fusiform gyrus & -45 & -66 & -15 & 5.24 & \\
\hline L & Posterior fusiform gyrus & -33 & -54 & -24 & 4.87 & \\
\hline L & Anterior fusiform gyrus & -45 & -48 & -21 & 4.16 & \\
\hline \multicolumn{7}{|c|}{$\begin{array}{l}\text { Repeated objects with } \\
\text { different view > same view }\end{array}$} \\
\hline $\mathrm{R}$ & Anterior fusiform gyrus & 42 & -45 & -30 & 4.25 & \\
\hline $\mathrm{R}$ & Posterior fusiform gyrus & 45 & -57 & -24 & 3.6 & * \\
\hline R & Posterior intraparietal sulcus & 36 & -78 & 33 & 4.1 & \\
\hline R & Parieto-occipital junction & 42 & -78 & 15 & 4.09 & \\
\hline $\mathrm{R}$ & Superior occipital gyrus & 51 & -69 & 18 & 3.54 & $*$ \\
\hline \multicolumn{7}{|c|}{$\begin{array}{l}\text { New objects > repeated objects with } \\
\text { different view and different size }\end{array}$} \\
\hline L & Posterior fusiform gyrus & -45 & -63 & -15 & 3.68 & \\
\hline L & Anterior fusiform gyrus & -39 & -39 & -24 & 3.67 & $*$ \\
\hline
\end{tabular}

Activation peaks significant at $P<0.05$ corrected for multiple comparisons, except for $* P<0.001$ uncorrected. 
a

Different > same view (rep. objects)
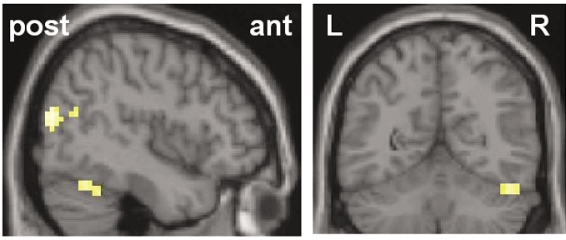

d

Different view (rep.) > new objects

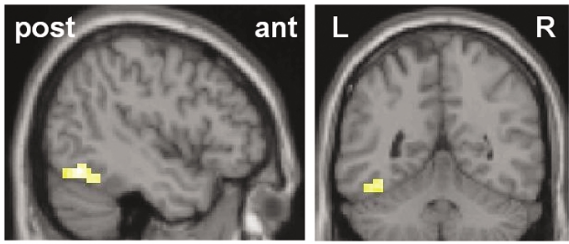

Different > same view (rep. objects)

g

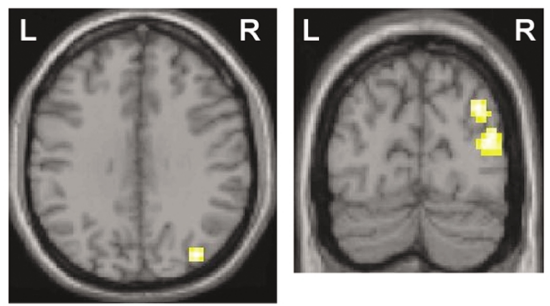

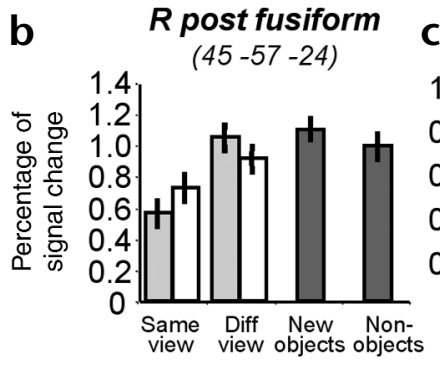
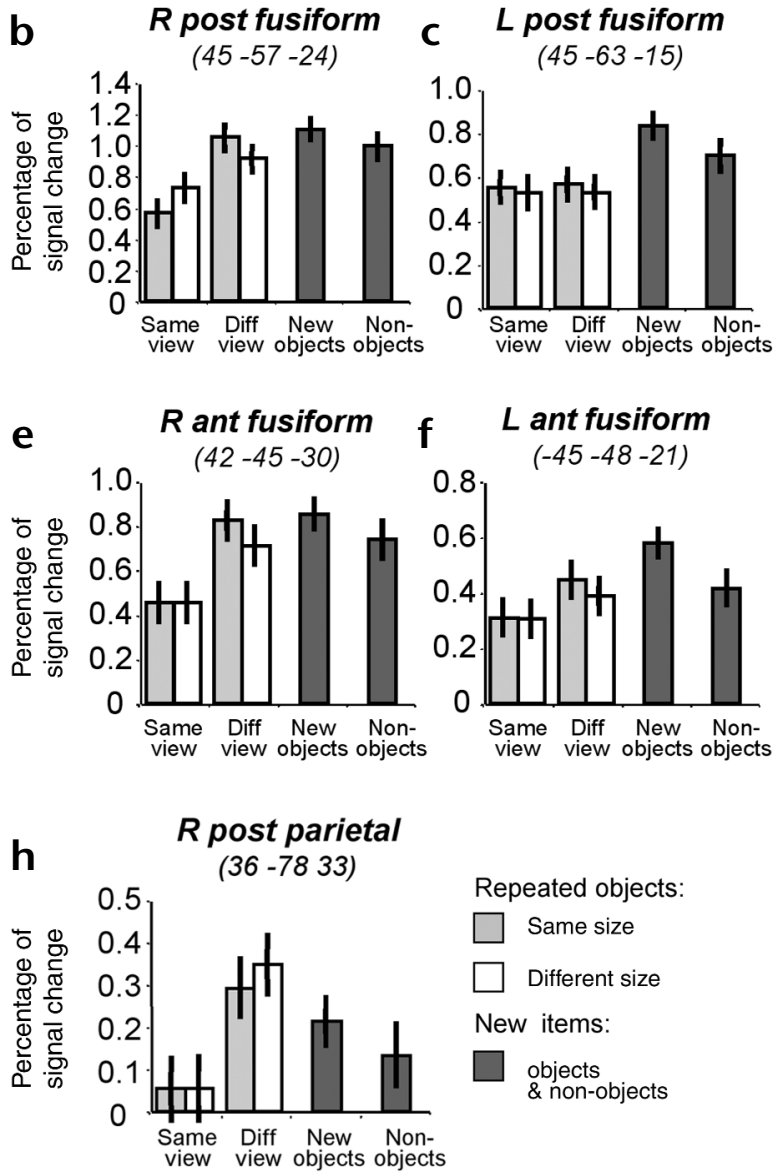

Fig. 3. Repetition priming effects in experiment 2. Group results are shown on a single-subject MRI scan (activated voxels $P<0.00 \mathrm{I}$ ) and plotted across conditions for main peaks of activity in homologous areas of right and left hemispheres (mean percent of signal change from baseline \pm s.e.m.). (a) Right fusiform and parieto-occipital regions show reduced activation by objects repeated with same versus different viewpoints. (b, e) This viewdependent priming in both anterior and posterior right fusiform was independent of object size changes. (d) Left fusiform regions show reduced activation by objects repeated with a different viewpoint, as compared to new objects. (c, $\mathbf{f}$ ) This view-independent and size-independent priming was found in both anterior and posterior left fusiform cortex. An apparent trend for a slight view-specific advantage in anterior left fusiform was not significant $(Z=1.62)$. ( $(\mathbf{g}, \mathbf{h})$ Posterior parietal regions showed not only less activation by objects repeated with the same view, but also greater activation by objects repeated with a different view, as compared to new objects.

fusiform were affected most by repetition of the real objects, though still responding to non-sense objects, whereas anterior fusiform regions were selectively engaged by meaningful stimuli (experiment 1) and showed generalization across changed visual appearance (experiment 2). The greater repetition effects for real versus non-sense objects in fusiform also corresponded with an overall enhanced fusiform activity for real objects,

\begin{tabular}{llrrrrr}
\hline \multicolumn{7}{l}{ Table 5. Retinotopic responses. } \\
\hline Side & Brain areas & \multicolumn{7}{c}{ Coordinates } & Z-score & \\
& & $x$ & $y$ & $z$ & & \\
All large > all small stimuli & & & & & \\
$\mathrm{R}$ & Upper striate cortex & 12 & -96 & 18 & 5.29 & \\
$\mathrm{R}$ & Lower striate cortex & 12 & $-8 \mathrm{I}$ & -12 & 4.32 & $*$ \\
$\mathrm{~L}$ & Upper striate cortex & -6 & -99 & 12 & 4.76 & \\
$\mathrm{~L}$ & Lower striate cortex & -21 & $-8 \mathrm{I}$ & -18 & 4.25 & $*$ \\
\hline
\end{tabular}

Activation peaks significant at $P<0.05$ corrected for multiple comparisons, except for $* P<0.001$ uncorrected. regardless of repetition (Table 3 and Fig. 2). This object selectivity probably did not result from differences in physical features between real and non-sense stimuli: heterogeneous sets were used for both, the result was found in both experiments (and for different item subsets within experiment 1), the sets did not differentially activate early visual cortex, and no area was activated more by non-sense than by real objects. The object selectivity may reflect an activation of pre-existing object representations, although we cannot entirely exclude the possibility that the object-decision task contributed to this effect.

Above these posterior-anterior differences, we found a left-right hemispheric asymmetry. Right fusiform responses to repetition priming were view dependent, showing greater decreases with the same viewpoint compared with different viewpoints. Changes in size did not matter. By contrast, priming in the left fusiform generalized across viewpoint as well as size (experiment 2), indicating that abstract, view-independent representations were involved. This view-invariant priming for meaningful objects in left fusiform was evidently visual, being independent of lexico-semantic factors (such as name 


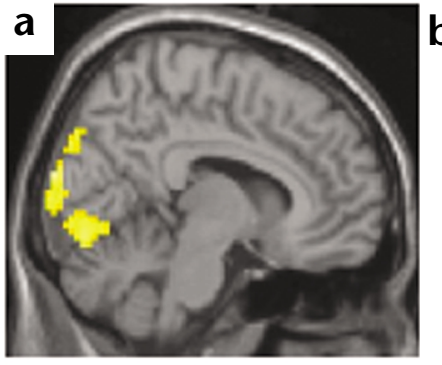

\section{b}

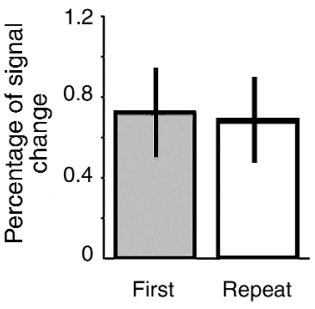

repetition) that affected only left inferior frontal cortex (experiment 1). Future studies may confirm that these results reflect priming of object recognition per se, unrelated to repetition of the task itself (for example, by using different tasks for experiments 1 and 2).

Our findings extend earlier imaging studies of object-repetition priming that broadly distinguished perceptual from conceptual ${ }^{23,24,26,28}$ or lexical-phonological ${ }^{29}$ processes in temporal and frontal regions. Here, we show that perceptual visual representations in temporal cortex can be fractionated into distinct neuronal components with different selectivity. In support of proposals that some priming effects depend on existing representations in the recognition system ${ }^{25,30}$, non-sense $3 \mathrm{D}$ objects (that were structurally possible) produced only weak repetition effects in lateral occipital and posterior temporal regions. Although this is consistent with the recently ascribed role of the 'lateral occipital complex' (LOC) in processing both familiar and unfamiliar shapes ${ }^{32-34,38,41}$, our data indicate that this region probably comprises distinct dorsal and ventral components ${ }^{31,42}$. Responses of the lateral occipital cortex (proper) to real versus non-sense objects were indistinguishable, but inferior temporal and fusiform gyri showed stronger responses and repetition decreases for real objects.

Stimulus size did not alter repetition effects in lateral occipital and ventral temporal areas, although the size differences produced substantial effects in early retinotopic cortex. This suggests that shape representation in these former areas is largely scale invariant. Other reports using fMRI adaptation (with blocks in which the same image repeats immediately and predictably, unlike here) showed that responses in LOC are invariant to changes in stimulus size, position and contour cues $^{31-33,41}$. These areas may be critical for extracting coherent shapes or bounded surfaces, rather than for recognizing objects per se. Previous studies did not, however, find LOC adaptation that was invariant to viewpoint ${ }^{31}$. Using wholebrain imaging, in a long-term repetition rather than adaptation design, we were able to demonstrate distinct view-dependent versus view-specific visual priming effects relating to object recognition in the human visual cortex.

Our most critical finding was that changes in viewpoint produced differential repetition effects for real objects in left versus

Fig. 5. Summary of main activation foci in experiments I and 2 (group results, $P<0.001$ ). Red areas showed common repetition-priming decreases for both real and non-sense objects (experiment I). Yellow areas showed greater repetition decreases for real objects repeated with the same view than with a different view (experiment 2). Blue areas showed repetition decreases for real objects previously exposed with a different view and different size, as compared with new real objects (experiment 2). Green areas showed selective repetition decreases for real objects but not non-sense objects (experiment I).
Fig. 4. Retinotopic effects were due to object size (group results, $P<$ 0.001 ). (a) Regions around the calcarine sulcus, representing the peripheral visual field in primary visual cortex, showed greater activation by larger objects but no repetition priming effect (both experiments). (b) First versus second presentation of the same items in experiment $\mathrm{I}$.

right fusiform regions. Right fusiform showed greater primingrelated decreases with repetition of the same viewpoint, irrespective of size, suggesting that neuronal populations in that region encode specific views of objects in a size-invariant manner. By contrast, homologous areas in left fusiform showed primingrelated decreases regardless of viewpoint, indicating neurons with greater object constancy across changes in inputs. Our data not only show that the visual system forms some view-specific representations even for familiar objects ${ }^{3,5,15,40,43}$, but also provide new evidence that other neuronal populations represent familiar objects in a more view-invariant form ${ }^{1,2,6,8}$, at least when the same crucial parts are maintained across view changes ${ }^{2,8,9}$. Future studies could adapt our design to test invariance across more radical changes in view ${ }^{2}$.

View-specific ${ }^{3,4,40}$ and view-invariant ${ }^{1,6,9}$ accounts of object recognition have often been regarded as mutually exclusive, but our new imaging data indicate that both may apply (to different neuronal populations). This corresponds with our behavioral data and previous behavioral studies ${ }^{2,8,44}$, indicating both view-dependent and view-independent benefits in object priming and learning. Finally, the hemispheric specialization we found for viewpoint specificity versus viewpoint independence is in line with recent behavioral visual-field effects for these forms of priming ${ }^{44}$.

It is important to note that the view-independent left fusiform responses in our study were item specific, and not attributable to semantic or name repetition ${ }^{24,28}$. This contrasts with fMRI findings of some fusiform asymmetry in object priming $^{28}$, where image-specific effects were found bilaterally and generalization to same-category exemplars was found in the left hemisphere. If pairs of exemplars were visually more similar in that study ${ }^{28}$ than in ours, left fusiform priming might reflect some sharing of characteristic visual features between objects of the same kind, rather than more abstract categorical factors. Left fusiform neurons might code for view-independent conjunctions of characteristic features ${ }^{1,2}$, whereas right fusiform might represent complex features in a view-specific (but scaleinvariant) holistic manner ${ }^{15,43,45}$. Such hemispheric specialization could allow the brain to use distinct types of representation conjointly ${ }^{11,44}$; it might even underlie the development of lateralized subsystems for face (holistic) and word (part-based) processing, but with bilateral object processing (both holistic

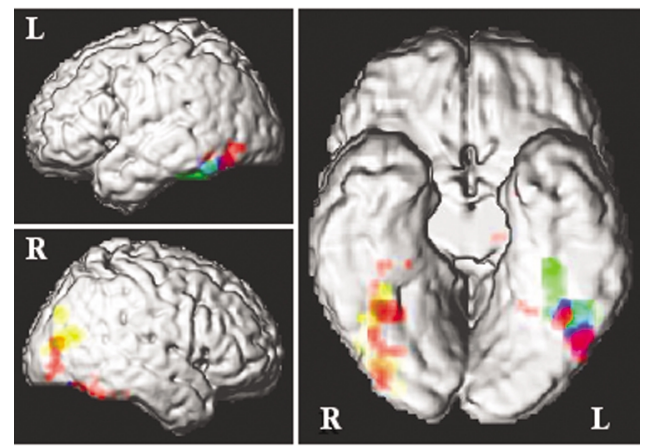


and part-based), as suggested by neuropsychological observations in brain-damaged patients ${ }^{10,11,46}$. View-independent processing in the left hemisphere could also result, in principle, from convergence of view-dependent representations from the same or opposite (right) hemisphere.

In conclusion, we used repetition-priming in event-related fMRI to assess multiple stages of object processing, from representations insensitive to previous knowledge in lateral occipital cortex, to size-invariant but view-dependent representations in right fusiform versus representations generalizing across both size and view in left fusiform, and finally lexico-semantic stages in left frontal cortex. Our study extends earlier work ${ }^{31,33}$ that showed short-term adaptation to the special case of immediate repetitions of the same image ${ }^{35,36}$, but found no viewpoint-independence in the selected regions of interest. By targeting neuronal populations that sustain object representations and long-term perceptual learning, we avoided potential attentional confounds and established direct relationships with behavioral priming.

Our results provide new insights into the human visual recognition system, revealing some similarities with monkey neurophysiology $15,47,48$ and some cortical asymmetries and lexico-semantic processes that may be unique to humans ${ }^{11,46}$. Moreover, our findings suggest that view-dependent and viewinvariant accounts of object recognition ${ }^{1-5,40,43}$ should be reconciled, as both may exist ${ }^{44}$ in distinct and differentially lateralized brain systems.

\section{Methods}

Subjects. Twelve neurologically healthy subjects ( 7 females and 5 males, mean age 27, range 19-39, all right-handed) gave informed consent according to procedures approved by the National Hospital and Institute of Neurology Ethics Committee. The two experiments were carried out in two separate scanning runs during a single session for each participant.

Stimuli. In experiment 1 , grayscale photographs of 80 different real objects (all man-made artifacts; no faces, houses or landscapes) and computerized pictures of 40 different non-sense objects (Fig. 1) were shown sequentially and repeated once after a pseudo-random lag (total 240 trials). To minimize the confound of repetition and time elapsed, the lags between the first and second occurrence of each stimulus were systematically distributed within either a relatively short (1-20 intervening stimuli) or long range (30-120), with more short than long lags, so that overall trial-ranks largely overlapped for first and second presentations (respectively, range 1-232, median 100; range 5-240, median 120). Pairs of real objects from the same category (for example, two distinct chairs) were divided into two sets (A and B), with all items from set $B$ appearing later than an item from set $A$ with the same name (mean $\operatorname{lag} 28 \pm$ s.d. 18).

In experiment 2 , the same 80 real objects as in experiment 1 were shown again, with either the same or different size (half each, with either $50 \%$ size increases or decreases, in equal number), and with either the same or different viewpoint (half each, with $45^{\circ}$ rotation of the camera view angle ${ }^{8}$ ). These were randomly intermixed with 40 new real objects and 40 new non-sense objects (matched to repeated objects in terms of size) that the subjects had not seen before. The mean pixelwise change ${ }^{31}$ between pairs of photographs was calculated as the rootmean-square difference in bitmap gray-level, scaled to have a possible maximum of 1 . This index included the common background, which comprised $73 \%$ of pixels on average. The indexed change was $0.104 \pm 0.041$ between photographs of a particular object repeated with different size but same view $(0.009$ when photographs were first normalized to their mean intensity); $0.103 \pm 0.034$ for same size but different views ( 0.008 normalized); and $0.121 \pm 0.041$ for exemplars from the same semantic category ( 0.012 normalized). Mean pixelchange did not differ for size changes versus view changes, but was greater $(P<0.0001)$ for same-category exemplars. The latter was similar to mean changes between two unrelated photographs $(0.132 \pm 0.047$, normalized 0.013$)$. All items were randomized and counterbalanced between conditions and experiments across subjects. As a result, the mean proportion of equivalent stimuli in the old or new category across subjects was $58 \% \pm 3 \%$ in experiment 2 (not different from chance, $\left.\chi^{2}(1)=1.28\right)$. Data from experiment 2 were lost for one subject because of technical failure.

Each item was preceded by a fixation cross for $500 \mathrm{~ms}$ and presented centrally for $500 \mathrm{~ms}$ (mean visual angle $5^{\circ}$ or $10^{\circ}$ for small or large stimuli), with a mean stimulus onset asynchrony of $4.5 \mathrm{~s}$ (randomly jittered between 3.6 and $11.0 \mathrm{~s}$ ).

Image acquisition. MRI data were acquired on a 2 Tesla Siemens (Erlangen, Germany) VISION system equipped with a head volume coil. Structural images were acquired with a T1-weighted sequence and functional images with a gradient echo-planar $\mathrm{T} 2{ }^{*}$ sequence using BOLD (blood oxygenation level-dependent) contrast. Each functional image comprised 30 transverse slices $(2 \mathrm{~mm}$ thickness, $2 \mathrm{~mm}$ gap, $64 \times 64,3 \times 3 \mathrm{~mm}^{2}$ pixels, $\mathrm{TE}=40 \mathrm{~ms}$ ) covering the whole brain. A total of 488 functional volumes in experiment 1 and 295 volumes in experiment 2 were acquired continuously with an effective repetition time (TR) of $2.28 \mathrm{~s}$.

Data were analyzed using the general linear model ${ }^{49}$ for eventrelated designs using SPM99 software (Wellcome Dept. of Cognitive Neurology, London, UK; http://fil.ion.ucl.ac.uk/spm). After all scans were realigned, normalized, time-corrected, and spatially smoothed by an $8 \mathrm{~mm}$ FWHM (full-width half-maximum) Gaussian kernel, a high-pass frequency filter (cutoff $120 \mathrm{~s}$ ) and corrections for auto-correlation between scans were applied to the time series. Individual events were modeled by a standard synthetic hemodynamic response function and its temporal derivative.

Seven event types were defined in each experiment, including the 6 critical experimental stimulus conditions (see above) with correct responses, plus any trials with incorrect responses. Movement parameters derived from realignment corrections ( 3 translations, 3 rotations) were also entered as covariates of no interest. We used the general linear model to generate parameter estimates of event-related activity at each voxel, for each condition and each subject. Statistical parametric maps of the $t$-statistic (SPM $\{t\}$ ) generated from linear contrasts between different conditions were transformed to a normal distribution $(\operatorname{SPM}\{Z\})$. SPM thresholds were set at $P=0.05$, corrected for multiple comparisons across all voxels showing a main effect of repetition in each experiment (at $P<0.001)^{50}$. Additional activations are reported at an uncorrected threshold of $P<0.001$ for descriptive purposes. Average group results were confirmed by secondary analyses with individual subjects as a random factor where indicated. In separate analyses, the effect of RTs on fMRI activity was examined using independent regressors that modeled a linear parametric modulation of the standard hemodynamic function by the RT, for each event in each subject (mean corrected), and two dichotomous event types (real or non-sense objects; first or second presentation). The effects of stimulus size were examined using four main event types (large or small, real or non-sense objects).

Note: Supplementary information is available on the Nature Neuroscience website.

\section{Acknowledgments}

We thank R. Ellis, M. Tucker and M. Tarr for some of the stimuli, and the radiographers at the Functional Imaging Laboratory for technical assistance. This work was supported by Wellcome Programme grants to R.J.D. and J.D., a Wellcome Fellowship to R.N.H. and a Medical Research Council (UK) Cooperative Grant for 'Analysis of cognitive impairment and imaging of cognition' at University College London. J.D. holds a Royal Society-Wolfson Research Merit Award.

\section{Competing interests statement}

The authors declare that they have no competing financial interests. 


\section{ReCeIVED 17 December 2001; ACCEPTED 28 February 2002}

1. Biederman, I. Recognition-by-components: a theory of human image understanding. Psychol. Rev. 94, 115-147 (1987).

2. Biederman, I. \& Gerhardstein, P. C. Recognizing depth-rotated objects: evidence and conditions for three-dimensional viewpoint invariance. J. Exp. Psychol. Hum. Percept. Perform. 19, 1162-1182 (1993).

3. Bulthoff, H. H., Edelman, S. Y. \& Tarr, M. J. How are three-dimensional objects represented in the brain? Cereb. Cortex 5, 247-260 (1995).

4. Tarr, M. J. \& Bulthoff, H. H. Image-based object recognition in man, monkey and machine. Cognition 67, 1-20 (1998).

5. Wallis, G. \& Bulthoff, H. Learning to recognize objects. Trends Cogn. Sci. 3, 22-31 (1999).

6. Marr, D. Vision (W. H. Freeman, San Francisco, 1982).

7. Tarr, M. J., Williams, P., Hayward, W. G. \& Gauthier, I. Three-dimensional object recognition is viewpoint dependent. Nat. Neurosci. 1, 275-277 (1998).

8. Ellis, R., Allport, D. A., Humphreys, G. W. \& Collis, J. Varieties of object constancy. Q. J. Exp. Psychol. A 41, 775-796 (1989).

9. Biederman, I. \& Bar, M. One-shot viewpoint invariance in matching novel objects. Vision Res. 39, 2885-2899 (1999).

10. Warrington, E. K. \& Taylor, A. M. Two categorical stages of object recognition. Perception 7, 695-705 (1978).

11. Grüsser, O. J. \& Landis, T. Visual agnosia and other disturbances of visual perception and cognition (ed. Cronly-Dillon, J. R.) (MacMillan, London, 1991).

12. Turnbull, O. H., Carey, D. P. \& McCarthy, R. A. The neuropsychology of object constancy. J. Int. Neuropsychol. Soc. 3, 288-298 (1997).

13. Lueschow, A., Miller, E. K. \& Desimone, R. Inferior temporal mechanisms for invariant object recognition. Cereb. Cortex 4, 523-531 (1994).

14. Booth, M. C. \& Rolls, E. T. View-invariant representations of familiar objects by neurons in the inferior temporal visual cortex. Cereb. Cortex 8, 510-523 (1998).

15. Logothetis, N. K., Pauls, J., Bulthoff, H. H. \& Poggio, T. View-dependent object recognition by monkeys. Curr. Biol. 4, 401-414 (1994).

16. Ashbridge, E., Perrett, D. I., Oram, M. W. \& Jellema, T. Effect of image orientation and size on object recognition: responses of single units in the macaque monkey temporal cortex. Cognit. Neuropsychol. 17, 13-34 (2000).

17. Schacter, D. L. \& Buckner, R. L. Priming and the brain. Neuron 20, 185-195 (1998).

18. Biederman, I. \& Cooper, E. E. Evidence for complete translational and reflectional invariance in visual object priming. Perception 20, 585-593 (1991).

19. Furmanski, C. S. \& Engel, S. A. Perceptual learning in object recognition: object specificity and size invariance. Vision Res. 40, 473-484 (2000).

20. Fiser, J. \& Biederman, I. Invariance of long-term visual priming to scale, reflection, translation, and hemisphere. Vision Res. 41, 221-234 (2001).

21. Cave, C. B., Bost, P. R. \& Cobb, R. E. Effects of color and pattern on implicit and explicit picture memory. J. Exp. Psychol. Learn. Mem. Cogn. 22, 639-653 (1996).

22. Desimone, R. Neural mechanisms for visual memory and their role in attention. Proc. Natl. Acad. Sci. USA 93, 13494-13499 (1996).

23. Blaxton, T. A. et al. Functional mapping of human memory using PET: comparisons of conceptual and perceptual tasks. Can. J. Exp. Psychol. 50, 42-56 (1996).

24. Buckner, R. L., Koutstaal, W., Schacter, D. L. \& Rosen, B. R. Functional MRI evidence for a role of frontal and inferior temporal cortex in amodal components of priming. Brain 3, 620-640 (2000).

25. Henson, R., Shallice, T. \& Dolan, R. Neuroimaging evidence for dissociable forms of repetition priming. Science 287, 1269-1272 (2000).

26. Buckner, R. L. et al. Functional-anatomic correlates of object priming in humans revealed by rapid presentation event-related fMRI. Neuron 20, 285-296 (1998).

27. James, T. W., Humphrey, G. K., Gati, J. S., Menon, R. S. \& Goodale, M. A. Repetition priming and the time course of object recognition: an fMRI study. Neuroreport 10, 1019-1023 (1999).

28. Koutstaal, W. et al. Perceptual specificity in visual object priming: functional magnetic resonance imaging evidence for a laterality difference in fusiform cortex. Neuropsychologia 39, 184-199 (2001).

29. van Turennout, M., Ellmore, T. \& Martin, A. Long-lasting cortical plasticity in the object naming system. Nat. Neurosci 3, 1329-1334 (2000).

30. Schacter, D. L. et al. Brain regions associated with retrieval of structurally coherent visual information. Nature 376, 587-590 (1995).

31. Kourtzi, Z. \& Kanwisher, N. Cortical regions involved in perceiving object shape. J. Neurosci. 20, 3310-3318 (2000).

32. Kourtzi, Z. \& Kanwisher, N. Representation of perceived object shape by the human lateral occipital complex. Science 293, 1506-1509 (2001).

33. Grill-Spector, K. et al. Differential processing of objects under various viewing conditions in the human lateral occipital complex. Neuron 24, 187-203 (1999).

34. Grill-Spector, K., Kourtzi, Z. \& Kanwisher, N. The lateral occipital complex and its role in object recognition. Vision Res. 41, 1409-1422 (2001).

35. Bentin, S. \& Moscovitch, M. The time course of repetition effects for words and unfamiliar faces. J. Exp. Psychol. Gen. 117, 148-60 (1988).

36. Nagy, M. E. \& Rugg, M. D. Modulation of event-related potentials by word repetition: the effects of inter-item lag. Psychophysiology 26, 431-436 (1989).

37. Friston, K. J., Holmes, A. P., Price, C. J., Buchel, C. \& Worsley, K. J. Multisubject fMRI studies and conjunction analyses. Neuroimage 10, 385-396 (1999).

38. Malach, R. et al. Object-related activity revealed by functional magnetic resonance imaging in human occipital cortex. Proc. Natl. Acad. Sci. USA 92, 8135-8139 (1995).

39. Wagner, A. D., Koutstaal, W., Maril, A., Schacter, D. L. \& Buckner, R. L. Taskspecific repetition priming in left inferior prefrontal cortex. Cereb. Cortex 10, 1176-1184 (2000).

40. Poggio, T. \& Edelman, S. A network that learns to recognize threedimensional objects. Nature 343, 263-266 (1990).

41. Grill-Spector, K., Kushnir, T., Edelman, S., Itzchak, Y. \& Malach, R. Cueinvariant activation in object-related areas of the human occipital lobe. Neuron 21, 191-202 (1998).

42. Lerner, Y., Hendler, T., Ben-Bashat, D., Harel, M. \& Malach, R. A hierarchical axis of object processing stages in the human visual cortex. Cereb. Cortex 11, 287-297 (2001).

43. Riesenhuber, M. \& Poggio, T. Hierarchical models of object recognition in cortex. Nat Neurosci 2, 1019-1025 (1999).

44. Burgund, E. D. \& Marsolek, C. J. Viewpoint-invariant and viewpointdependent object recognition in dissociable neural subsystems. Psychonom. Bull. Rev. 7, 480-489 (2000).

45. Tsunoda, K., Yamane, Y., Nishizaki, M. \& Tanifuji, M. Complex objects are represented in macaque inferotemporal cortex by the combination of feature columns. Nat. Neurosci. 4, 832-838 (2001).

46. Farah, M. J. Visual Agnosia: Disorders of Object Recognition and What They Tell Us About Normal Vision (MIT Press, Cambridge, Massachusetts, 1990).

47. Wallis, G. \& Rolls, E. T. Invariant face and object recognition in the visual system. Prog. Neurobiol. 51, 167-194 (1997).

48. Tanaka, K. Inferotemporal cortex and object vision. Annu. Rev. Neurosci. 19, 109-139 (1996).

49. Friston, K. J. et al. Statistical parametric maps in functional imaging: a general linear approach. Hum. Brain Mapp. 2, 189-210 (1995).

50. Worsley, K. J. et al. A unified statistical approach for determining significant signals in images of cerebral activation. Hum. Brain Mapp. 4, 58-73 (1996). 